\title{
The crust of rotating strange quark stars
}

\author{
J. L. Zdunik ${ }^{1}$, P. Haensel ${ }^{1,2}$, and E. Gourgoulhon ${ }^{2}$ \\ 1 N. Copernicus Astronomical Center, Polish Academy of Sciences, Bartycka 18, 00-716 Warszawa, Poland \\ 2 Département d'Astrophysique Relativiste et de Cosmologie - UMR 8629 du CNRS, Observatoire de Paris, \\ 92195 Meudon Cedex, France \\ e-mail: jlz@camk.edu.pl; haensel@camk.edu.pl; Eric.Gourgoulhon@obspm.fr
}

Received 12 January 2001 / Accepted 9 March 2001

\begin{abstract}
Calculations of the properties of rotating strange stars with crusts are performed within the framework of general relativity. We employ an equation of state (EOS) of strange quark matter based on the MIT Bag Model with massive strange quarks and lowest order QCD interactions. The crust is described by the BPS equation of state. A significant increase of the stellar radius is found close to the Keplerian (mass-shedding limit) configuration. This leads to the disappearance of the gap between the stellar surface and the innermost stable circular orbit (ISCO) at very high rotation rates, for a rather broad range of stellar masses. The Keplerian configuration for the strange star with crust corresponds to values of $J, T / W, P_{\mathrm{ISCO}}=1 / \nu_{\mathrm{ISCO}}$ which are about $20 \%$ smaller than in the case of bare strange stars. Because the Keplerian configuration is achieved due to the increase of the stellar oblateness, the Keplerian frequency (of the rotation) remains almost unaltered. The lack of the gap close to the Keplerian rotation could imply a more stringent limit on $\nu_{\mathrm{ISCO}}$, if the existence of such a gap is supported by observations, as in the source $4 \mathrm{U} 1820-30$ with the upper QPO frequency $1.07 \mathrm{kHz}$. If such a constraint is taken into account (mandatory existence of a gap) the minimum $\nu_{\mathrm{ISCO}}$ is about $1 \mathrm{kHz}$ even with the extreme fine tuning of strange quark matter parameters. The minimum $\nu_{\mathrm{ISCO}}$ is then obtained for the non-rotating configuration with maximum allowable mass. The maximum frequency in the stable circular orbit around the strange star with a crust is smaller by about $100 \mathrm{~Hz}$ than in the case of a bare strange star. During the spin-down of a magnetized strange quark star with crust, the crust matter is absorbed in the equatorial region by the strange matter core. The deconfinement of absorbed crust matter is a strongly exothermic process, which would influence the cooling curve of this compact object.
\end{abstract}

Key words. dense matter - equation of state - stars: neutron - stars: rotation - stars: binaries: general

\section{Introduction}

Since the mid-1980, when these exotic compact stars were introduced (Witten 1984; first detailed models in Haensel et al. 1986; Alcock et al. 1986), the existence of strange quark stars in the Universe remains a matter of lively debate (for a review, see Madsen 2000). Recently, these hypothetical objects were invoked in the context of modeling compact X-ray and gamma-ray sources (Bombaci 1997; Cheng et al. 1998; Dai \& Lu 1998; Li et al. 1999). Simultaneously, several studies, in which exact 2-D general-relativistic calculations of stationary rigidly rotating configurations were performed, focused on the substantial difference in rapid rotation of strange quark stars and (normal) neutron stars. This feature stems from the basic difference in equations of state of these two types of compact superdense stars (Gourgoulhon et al. 1999; Stergioulas et al. 1999; Zdunik et al. 2000;

Send offprint requests to: J. L. Zdunik, e-mail: jlz@camk.edu.pl
Gondek-Rosińska et al. 2000). In particular, the difference in the distribution of matter in the rapidly rotating models was shown to imply significant difference in the outer spacetime. Specifically, qualitative differences in the properties of the innermost stable circular orbit (ISCO) around these two classes of rapidly rotating compact objects have been found (Stergioulas et al. 1999; Zdunik et al. 2000). The comparison of numerical results for the ISCO (in particular, the rotation frequency of plasma blobs on the ISCO, $\nu_{\mathrm{ISCO}}$ ) with observations of the $\mathrm{kHz}$ quasi-periodic oscillations (QPO) in some of the low-mass X-ray binaries (LMXB) was used to put constraints on the strange quark matter (SQM) models.

A strange star could be covered by a crust of normal matter, a possibility which is particularly natural in the case of the LMXB (or of a strange star which went through a LMXB epoch). The problem of formation and structure of a crust on an accreting strange star was studied by Haensel \& Zdunik (1991) (see also MiraldaEscudé et al. 1990). Because of its low mass, typically 
$\lesssim 10^{-5} M_{\odot}$, the effect of the crust on the exterior spacetime is negligible. However, due to its thickness, the crust determines the location of the stellar surface. As it has been shown in Zdunik et al. (2000), the presence of the crust could play an important role in strange stars rotating close to Keplerian (mass-shedding limit) frequency. Namely, the crust undergoes substantial inflation in the equatorial plane, leading to the disappearance of the gap between the ISCO and stellar surface. The crust mass, thickness and other macroscopic parameters depend only on the gravitational force exerted by the SQM core (which is determined by the core mass and radius), and on rotation frequency.

Preliminary, approximate studies of the crust on rotating strange stars have been performed by Glendenning \& Weber (1992), in the framework of Hartle's (1967) slow rotation approximation supplemented by the selfconsistency conditions allowing for determination of the stellar parameters close to the Keplerian frequencies $\nu_{\text {rot }} \simeq \nu_{\mathrm{K}}$. The results of this approach were exploited by Zdunik et al. (2000). They have shown that at some fixed total baryon mass, $M_{\mathrm{B}}$, of the strange star, the rotational increase of the equatorial radius, relative to the nonrotating case, is quadratic in $\nu_{\text {rot }} / \nu_{\mathrm{K}}$. As Zdunik et al. (2000) have found, the quadratic approximation described very precisely numerical results of Glendenning \& Weber (1992), up to $\nu_{\text {rot }}=\nu_{\mathrm{K}}$.

In the present paper we present results of exact (i.e. not based on the slow rotation approximation) 2-D calculations of the structure of the crust on rotating strange star. We show that the quadratic approximation for $R_{\text {eq }}\left(\nu_{\text {rot }}\right)-R(0), M_{\mathrm{B}, \text { crust }}\left(\nu_{\text {rot }}\right)-M_{\mathrm{B}, \text { crust }}(0)$ is very precise for $\nu_{\text {rot }} \lesssim 500 \mathrm{~Hz}$, becomes less precise with a further increase of $\nu_{\text {rot }}$, and badly underestimates the increase of $R_{\text {eq }}$ and $M_{\mathrm{B} \text {,crust }}$ for $\nu_{\text {rot }} \simeq \nu_{\mathrm{K}}$. Consequently, the presence of the solid crust substantially changes the parameters of the Keplerian configuration, and the properties of the ISCO for $\nu_{\text {rot }} \simeq \nu_{\mathrm{K}}$.

Strange quark stars with a crust could possess a strong magnetic field, and their magnetosphere may be similar to that of a neutron star. Therefore, their rapid rotation might be expected to be slowed down by magnetic braking. Slowing down of rotation would imply a compression of the crust matter in the equatorial band. As the density at the crust bottom cannot exceed the critical value $\rho_{\mathrm{b}}$ at which crust matter becomes unstable with respect to irreversible absorption by the SQM (due to neutron drip or quantum tunneling of nuclei through the Coulomb barrier), the slowing down of rotation is accompanied by a decrease in the baryon mass of the crust $M_{\mathrm{B} \text {,crust }}$, and a heating due to energy release accompanying absorption and deconfinement of baryons (deconfinement heating). We derive analytical formulae expressing the rate of deconfinement heating as a function of $\dot{\nu}_{\text {rot }}$ and $\nu_{\text {rot }}$. Deconfinement heating, as well as heating due to non-equilibrium weak interaction processes in SQM (Cheng \& Dai 1996) will influence the cooling curve of rotating magnetized strange quark stars with a crust (Yuan \& Zhang 1999).
In Sect. 2 we briefly present the equation of state SQM and the method for constructing relativistic 2-D models of stationary rotating strange quark stars with crust. Numerical results for the parameters of rapidly rotating strange stars, including the ISCOs, are presented in Sect. 3. Exact numerical results for the crust on rotating strange stars are fitted using simple, convenient, analytical expressions. The deconfinement heating of spinningdown strange quark stars with crusts is studied in Sect. 4. Finally, Sect. 5 contains discussion of our results and conclusions.

\section{Equation of state and calculation of rotating strange star models}

\subsection{Equation of state of strange quark matter}

The differences between the density profiles of a strange star and a neutron star result from the basic difference in the EOS of their interiors. In the case of a rapidly rotating compact object (situation relevant to LMXB), the differences between the matter distributions within a neutron star and a strange star may be expected to imply differences in the shape of rotating objects and in the spacetime exterior to the star, and in particular, differences in the properties of the ISCO.

Our EOS of strange matter, composed of massless $u$, d quarks, and massive s quarks, is based on the MIT Bag Model. It involves three basic parameters: the bag constant, $B$, the mass of the strange quarks, $m_{\mathrm{s}}$, and the QCD coupling constant, $\alpha_{\mathrm{c}}$ (Farhi \& Jaffe 1984; Haensel et al. 1986; Alcock et al. 1986). Our basic EOS corresponds to standard values of the Bag Model parameters for strange matter: $B=56 \mathrm{MeV} / \mathrm{fm}^{3}, m_{\mathrm{s}}=200 \mathrm{MeV} / \mathrm{c}^{2}$, and $\alpha_{\mathrm{c}}=$ 0.2 (Farhi \& Jaffe 1984; Haensel et al. 1986; Alcock et al. 1986). This EOS of strange quark matter will be hereafter referred to as the SQM1 one. It is the same as that used in Zdunik et al. (2000) and Zdunik \& Gourgoulhon (2001). It yields energy per unit baryon number at zero pressure $E_{0}=918.8 \mathrm{MeV}<E\left({ }^{56} \mathrm{Fe}\right)=930.4 \mathrm{MeV}$. For the SQM1 EOS the maximum allowable mass for static strange stars is $M_{\max }^{\text {stat }}=1.8 M_{\odot}$.

\subsection{Solid crust of strange quark star}

The crust in our model is described by the BPS model of dense matter below the neutron drip (Baym et al. 1970). Neutrons are absorbed by SQM, and therefore the density at the bottom of the crust, $\rho_{\mathrm{b}}$, cannot be higher than neutron drip density $\rho_{\mathrm{ND}} \simeq 4 \times 10^{11} \mathrm{~g} \mathrm{~cm}^{-3}$. A superstrong outward-directed electric field separates nuclei of the crust from the quark-plasma edge, which is necessary for the crust stability (Alcock et al. 1986). The gap between nuclei and SQM surface should be sufficiently large to prevent the absorption of nuclei by SQM via quantum Coulomb barrier penetration. If at $\rho_{\mathrm{ND}}$ the gap is sufficiently large and the Coulomb barrier is sufficiently high, then $\rho_{\mathrm{b}}=\rho_{\mathrm{ND}}$. Another possibility is that the gap and the 
Coulomb barrier at $\rho_{\mathrm{ND}}$ can be penetrated by nuclei, and then the stability conditions are reached at some lower value of $\rho_{\mathrm{b}}$. In such a case, both mass and thickness of the crust are lower than that corresponding to $\rho_{\mathrm{b}}=\rho_{\mathrm{ND}}$ (see, e.g., Huang \& Lu 1997). In what follows, we will assume that $\rho_{\mathrm{b}}=\rho_{\mathrm{ND}}$. The EOS of the crust is joined with that of the SQM core by requiring the continuity of pressure at the crust-SQM core interface.

\subsection{Calculation of models of stationary rotating strange stars with crust}

The general relativistic models of stationary rotating strange stars have been calculated by means of the code developed by Gourgoulhon et al. (1999), which relies on the multi-domain spectral method introduced by Bonazzola et al. (1998). With respect to the version of the code used in Gourgoulhon et al. (1999), Zdunik et al. (2000), Gondek-Rosińska et al. (2000), Zdunik \& Gourgoulhon (2001), we introduce a second domain inside the star to describe its crust, resulting in a total of four domains. Using the same notation as in Sect. 3.2 of Gourgoulhon et al. (1999), these four domains are: D1, which covers the strange quark interior; D2, which contains the crust; D3, which covers the strong field region outside the star (and where the ISCO is located) and D4, which extends to infinity, thanks to the compactification transformation $u=1 / r$. Following the technique explained in Bonazzola et al. (1998), the boundary between domains D1 and D2 is forced to coincide with the transition between the strange quark matter and the crust, so that the huge density discontinuity between the two regions (cf. Fig. 4) is located at the boundary between the computational domains D1 and D2 and therefore does not give rise to spurious oscillations (Gibbs phenomenon). The number of coefficients used in the spectral expansions (or equivalently number of grid points) is typically 33 in $r$ and 17 in $\theta$ in each of the four domains. The global numerical error is evaluated by means of the virial identities GRV2 (Bonazzola \& Gourgoulhon 1994) and GRV3 (Gourgoulhon \& Bonazzola 1994), this latter being a relativistic generalization of the classical virial theorem. For instance, for the configurations depicted in Figs. 3 and 4, the GRV2 (resp. GRV3) relative error is $6 \times 10^{-5}$ (resp. $\left.4 \times 10^{-4}\right)$. The radius and the frequency of the marginally stable orbit, $R_{\mathrm{ms}}$, and its frequency $\nu_{\mathrm{ms}}$ is determined in a standard way (see, e.g., Cook et al. 1994, for the equations to be solved).

Note that in all the results presented in this article, the equatorial radius of the star $R_{\text {eq }}$ is defined as the circumferential radius (i.e. the length of the equator, given by the spacetime metric, divided by $2 \pi$ ). In the nonrotating limit, this equatorial radius reduces to the surface value of the Schwarzschild coordinate $R$ employed in the TolmanOppenheimer-Volkoff equations. Note also that all nonequatorial coordinates (e.g. polar radius, Figs. 3 and 4) are obtained by rescaling of the radial quasi-isotropic co-

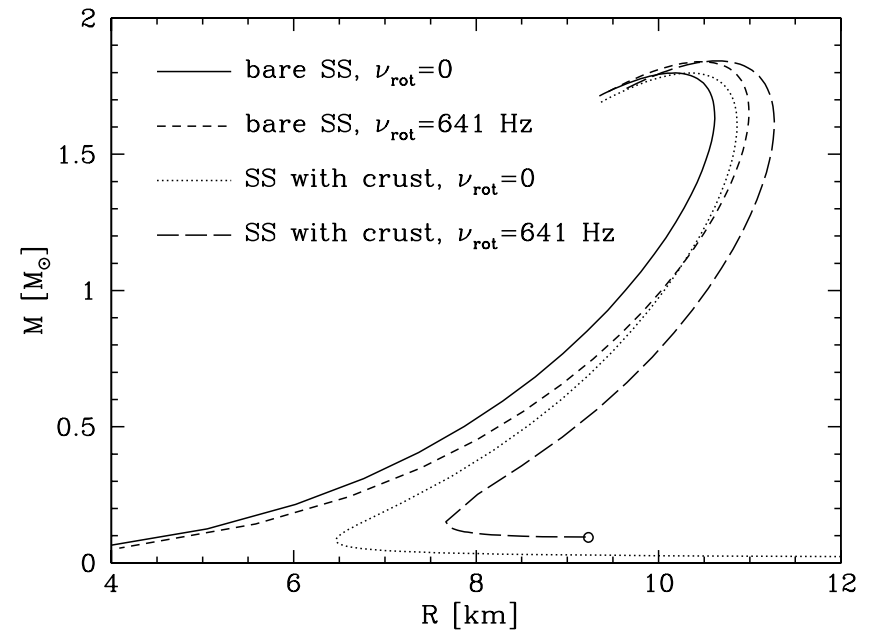

Fig. 1. Mass versus (circumferential) equatorial radius for nonrotating bare strange stars (SS, solid line), nonrotating SS with crust (dotted), and SS (bare and with crust) rotating with the maximum observed frequency $(641 \mathrm{~Hz})$. The short-dashed line corresponds to a rotating bare SS and the long-dashed one to a rotating SS with crust (the open circle marks the point at which $641 \mathrm{~Hz}$ is the Keplerian frequency).

ordinate $r$ (see the line element (6) in Gourgoulhon et al. 1999) by the factor $R_{\text {eq }} / r_{\text {eq }}$.

Let us consider a strange star, rotating at a frequency $\nu_{\text {rot }}$, with equatorial radius $R_{\text {eq }}$. If $R_{\mathrm{ms}}>R_{\text {eq }}$, then stable orbits exist for $r>R_{\mathrm{ms}}$; the ISCO then has the radius $R_{\mathrm{ms}}$ and the frequency $\nu_{\mathrm{ms}}$, and there is a gap of width $R_{\mathrm{ms}}-R_{\mathrm{eq}}$ between the ISCO and the strange star surface. However, if $R_{\mathrm{ms}}<R_{\mathrm{eq}}$, then $R_{\mathrm{ISCO}}=R_{\mathrm{eq}}$, $\nu_{\mathrm{ISCO}}=\nu_{\mathrm{orb}}\left(R_{\mathrm{eq}}\right)$; an accretion disk then extends down to the strange star surface (or, more precisely, joins the stellar surface via a boundary layer). It should be noted that the recent analysis of the observational data strongly supports the existence of the marginally stable orbit in the case of $4 \mathrm{U}$ 1820-30 (Kaaret et al. 1999).

\section{Results}

\subsection{Global stellar parameters}

In the present section we describe the global parameters of the rotating strange stars with a crust. We compare these results with those obtained for bare strange stars and with results of Glendenning \& Weber (1992).

In Fig. 1 we present the mass versus radius relation for nonrotating strange stars and for stars rotating with a period equal to the smallest measured period of pulsars (1.56 ms, which corresponds to a rotation frequency of $641 \mathrm{~Hz}$ ). The last point on the strange star (SS) with crust curve corresponds to the Keplerian frequency of this star, equal to $641 \mathrm{~Hz}$. Strange stars with smaller mass have thicker crusts and $\nu_{\mathrm{K}}<641 \mathrm{~Hz}$.

In the next figures we present global parameters of rotating strange stars (with and without crust) along some evolutionary sequences with fixed total baryon numbers, 


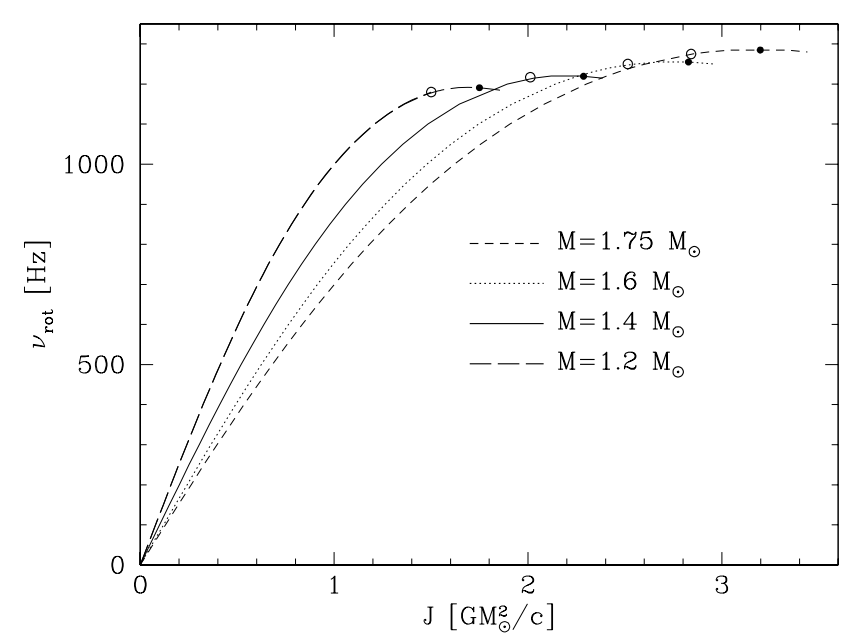

Fig. 2. Rotation frequency versus angular momentum of SS along constant baryon number sequences. Each sequence is labeled by the gravitational mass $M$ of its nonrotating configuration. The last points of these curves correspond to the Keplerian configurations for bare SS: filled dots: GlendenningWeber approximation of the rotating crust; open dots: exact calculations of the Keplerian frequency.

equal to those of nonrotating configurations of given gravitational masses $\left(M=1.2,1.4,1.6,1.75 M_{\odot}\right)$. Of course, due to the rotation, the actual gravitational mass of the star increases with increasing rotation frequency. It is the baryon mass $M_{\mathrm{B}}$ which is fixed for each sequence ("evolutionary sequence").

In these figures, the Keplerian configuration obtained in our exact calculations is marked by an open circle. For comparison, we also marked by filled dots the results of (Zdunik et al. 2000), obtained using the Glendenning \& Weber (1992) model for the crust on a rotating strange star. The last point of each curve corresponds to the Keplerian configuration for the bare strange star.

In Fig. 2 we present $\nu_{\text {rot }}$ as a function of the angular momentum of the star $J$. We see that for a bare strange star the Keplerian configuration is not achieved via the increase of $\nu_{\mathrm{rot}}$. On the contrary, close to this configuration there exists a region for which $\nu_{\text {rot }}$ is a slightly decreasing function of $J$ : this corresponds to spin up by the angular momentum loss. Therefore, $\nu_{\text {rot }}^{\max }>\nu_{\text {rot }}\left(J_{\max }\right) \equiv \nu_{\mathrm{K}}$.

This effect turns out to be suppressed by the existence of the crust. Although the crust does not change in practice the curve $\nu_{\text {rot }}(J)$, the radius of a rotating star with a crust is significantly larger, setting the maximum value of $J$ to be smaller by $\sim 20 \%$ than for a bare strange star. However, due to the flatness of the function $\nu_{\text {rot }}(J)$ for high $J$, the Keplerian frequency is nearly the same as in the case of bare SS.

The reason why $\nu_{\text {rot }}(J)$ does not increase close to $J_{\max }$ is explained in Fig. 5. The increase of $J$ results in flattening of the star ( $R_{\mathrm{eq}}$ increases, so $J$ increases without change in $\left.\nu_{\text {rot }}\right)$. In other words, the Keplerian configuration is achieved due to the increase in the equatorial radius related to the deformation of the star. We see that

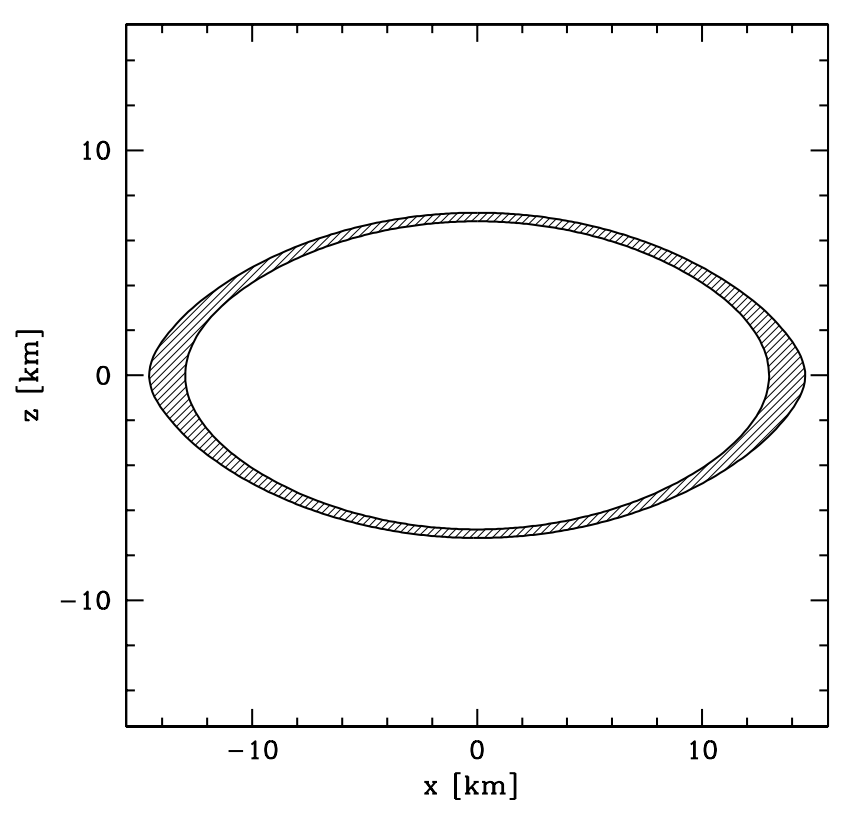

Fig. 3. The edge of the SQM core and the crust surface for a strange quark star with crust (baryon mass $M_{\mathrm{B}}=1.63 M_{\odot}$ ), rotating at $\nu_{\text {rot }}=1210 \mathrm{~Hz}$. The Keplerian frequency $\nu_{\mathrm{K}}=$ $1217 \mathrm{~Hz}$ (see Sect. 2.3 for the definition of the coordinates used in this plot).

the strange star with a crust is more oblate than bare SS for the same $J$. This is due to the fact that the crust is the most deformed part of the star. This effect is visualized in Figs. 3 and 4, in which we show the effect of rotation at $\nu_{\text {rot }}=1210 \mathrm{kHz}$ on the structure of a strange quark star of $M_{\mathrm{B}}=1.63 M_{\odot}$. As apparent in Fig. 3, the crust thickness at the equator is much greater than at the pole (about five times). Correspondingly, the density gradient in the polar ring of the crust is dramatically less steep than at the pole (see Fig. 4 ). Generally, the increase of $R_{\text {eq }} / R_{\text {pole }}$ with the total stellar angular momentum $J$ depends on the baryon mass of the star: the lower the value of $M_{\mathrm{B}}$, the steeper the increase of $R_{\text {eq }} / R_{\text {pole }}$ with increasing $J$. This is visualized in Fig. 10.

The global parameter which is relevant from the point of view of the stability of rapidly rotating stars with respect to spontaneous triaxial deformations is the ratio of kinetic to gravitational energy $T / W$ (Shapiro \& Zane 1998). In Fig. 6 we have plotted $T / W$ versus rotation frequency, for four evolutionary sequences of rotating strange stars with some fixed baryon number. The differences in the maximum value of $T / W$ between the cases of bare strange stars and strange stars with crust are quite significant. For strange stars with crust the maximum value $T / W \approx 0.18 \div 0.19$, which is more than $20 \%$ smaller than in the case of bare strange stars, with maximum $T / W \approx 0.25$.

\subsection{Innermost stable circular orbit}

Previous calculations suggested that the gap between the ISCO and the stellar surface exists for Keplerian 


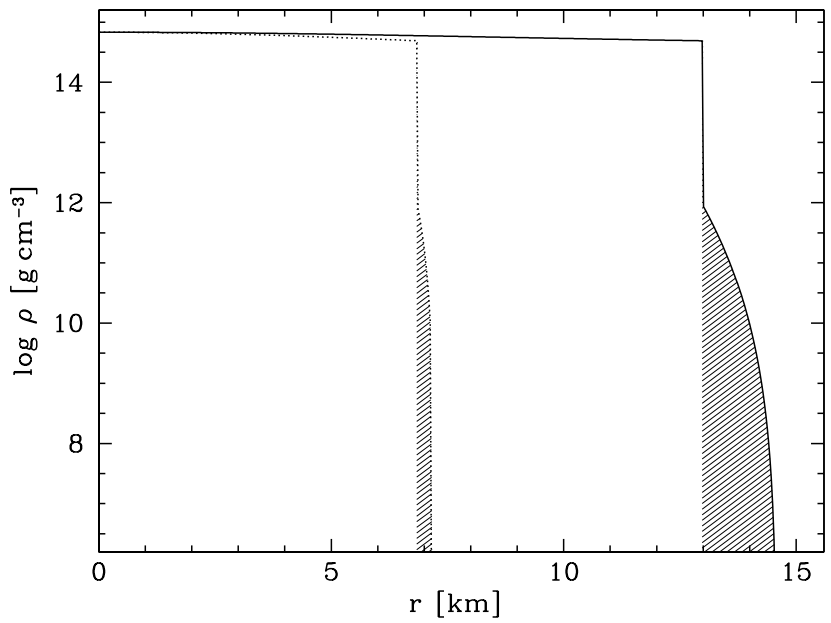

Fig. 4. Logarithm of density versus the radial coordinate (defined in Sect. 2.3) along the polar (dotted line) and equatorial (solid line) directions. The strange star model is the same as in Fig. 3. The shaded areas correspond to the crust.

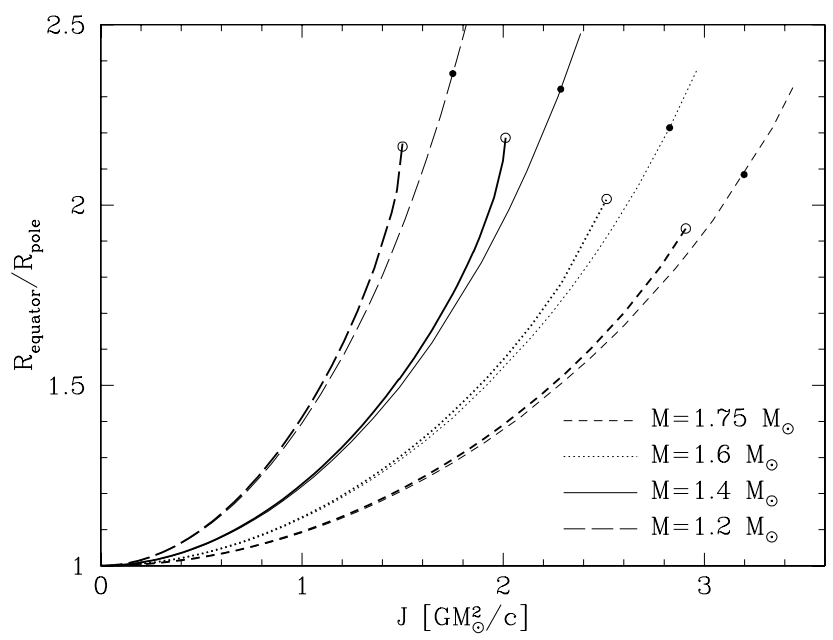

Fig. 5. Oblateness of the star presented as the ratio of the equatorial to polar radius, versus stellar angular momentum $J$, along some constant baryon number sequences. Each sequence is labeled by the gravitational mass $M$ of its non-rotating configuration. Thin lines correspond to bare strange stars.

configurations within a rather broad range of stellar masses (Stergioulas et al. 1999; Zdunik et al. 2000). Our calculations, presented in Fig. 7, indicate that for strange stars with crusts the gap exists only very close to the maximum baryon mass. For lower masses (even 1.6 $M_{\odot}$ for the SQM1 model for which $\left.M_{\max }^{\text {stat }}=1.8 M_{\odot}\right)$ there is no gap close to the Keplerian configuration. Thus if the observational data support the existence of the gap, the theoretical lower limit on $\nu_{\text {ISCO }}$ for rapidly rotating strange stars will be higher (asterisks in Fig. 7).

For bare strange stars the gap exists up to the Keplerian frequency. Even in the case of smaller mass stars for which $R_{\mathrm{ms}}<R_{\mathrm{eq}}$ for intermediate frequencies (e.g. $\left.M=1.2 M_{\odot}\right)$ the gap reappears at high rotation rates. In the extreme case of low mass bare strange stars (for which relativistic effects are negligible) the gap appears close to

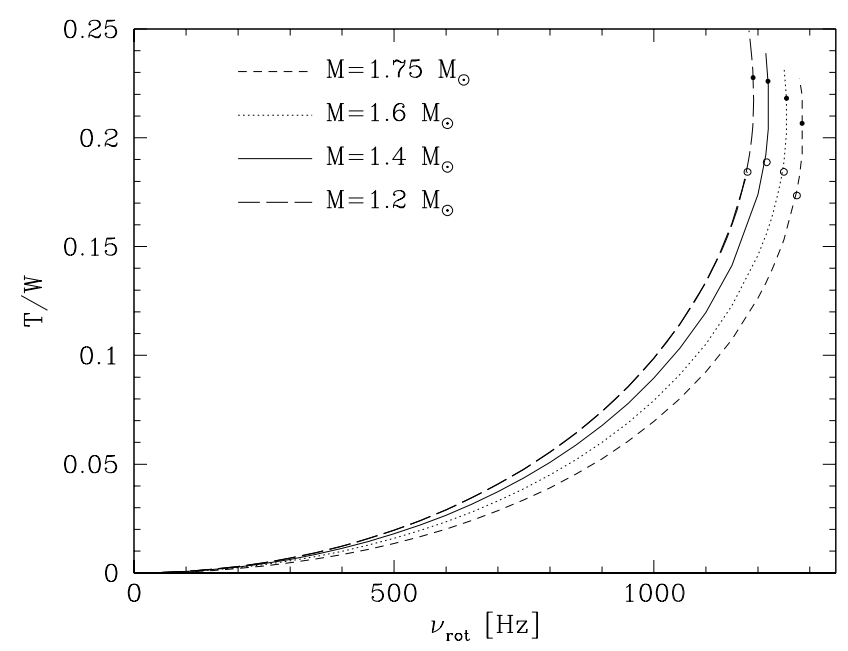

Fig. 6. $T / W$ ratio as a function of $\nu_{\text {rot }}$ along some constant baryon number sequences. Notations as in Fig. 5 .

Keplerian frequency due to the large oblateness of the star (Zdunik \& Gourgoulhon 2001). As a result, the frequency of the ISCO at the Keplerian limit is much smaller than for the nonrotating star (especially for low-mass stars).

Of course, the smaller the mass, the larger the difference between bare strange stars and strange stars with a crust, the size of the crust being a decreasing function of $M$. For low mass strange stars with crusts $\left(M<1.1 M_{\odot}\right)$ the gap between the surface and the marginally stable orbit vanishes independent of the rotation rate. In the intermediate mass range $\left(\sim 1.1 \div 1.3 M_{\odot}\right)$ the gap exists for a nonrotating star, then disappears for larger rotation rates and reappears at high frequencies. In this case the difference between a bare strange star and a star with a crust is clearly visible (Fig. 7). Due to the existence of the crust, the region of $\nu_{\text {rot }}$ in which the ISCO is determined by the stellar surface (i.e. $R_{\mathrm{ms}}<R_{\mathrm{eq}}$ ) is larger and thus the frequency $\nu_{\mathrm{ISCO}}$ is significantly smaller because the orbit of ISCO corresponds then to the equatorial radius and not to the mass of the star (as in the case of the marginally stable orbit). As a result, the maximum possible value of the frequency of the particle in a stable circular orbit around the strange star with crust (which is determined by the point $R_{\mathrm{ms}}=R_{\mathrm{eq}}$ ) is smaller by about $100 \mathrm{~Hz}$ than for bare SS.

For relatively massive strange stars with a crust $\left(\sim 1.3 \div 1.6 M_{\odot}\right)$, the gap exists for all rotation rates except those very close to $\nu_{\mathrm{K}}$. The condition $R_{\mathrm{ms}}=R_{\text {eq }}$ defines then the minimum ISCO frequency consistent with the constraint of the existence of the marginally stable orbit $\left(\sim 1.3 \mathrm{kHz}\right.$ for $1.2<M / M_{\odot}<1.6$ in the case of the SQM1 EOS, asterisks in Fig. 7).

Only for stars with a mass close to the maximum for nonrotating configurations $\left(\sim 1.6 \div 1.8 M_{\odot}\right)$ is the marginally stable orbit located above the stellar surface at Keplerian rotation rate.

As the Keplerian rotation corresponds to the minimum of $\nu_{\mathrm{ISCO}}$, the existence of the crust increases these values by $100 \div 200 \mathrm{~Hz}$ compared to bare strange stars. As one can 


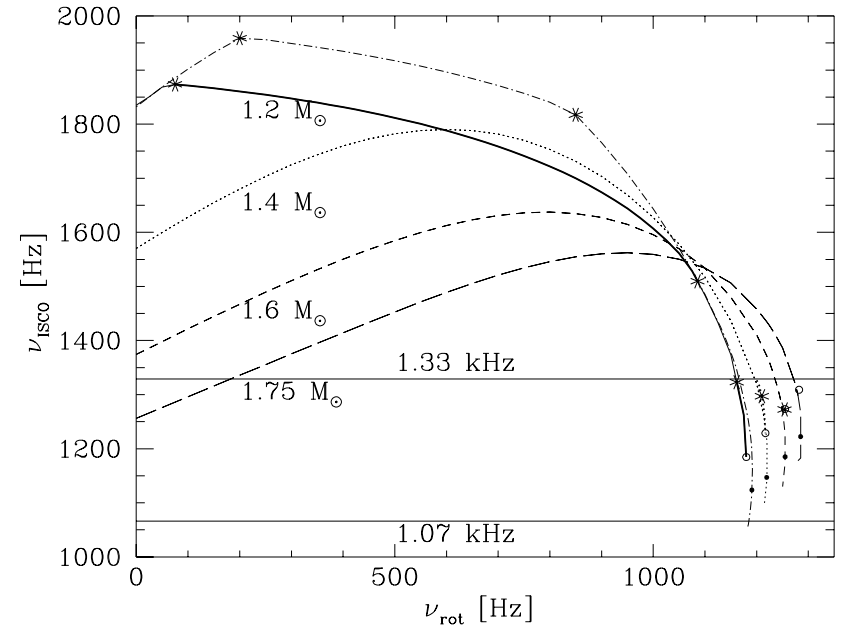

Fig. 7. Orbital frequency of the ISCO versus the rotation frequency of strange stars, for the SQM1 EOS of strange matter. Each curve corresponds to a fixed baryon mass, equal to that of a static strange star of gravitational mass indicated by a label. Along each curve, the angular momentum increases from $J=0$ (static configuration) to $J_{\max }$ (Keplerian limit). Open circles correspond to Keplerian configurations of strange stars with crust (filled circles - approximation of the crust based on the results of Glendenning \& Weber 1992). Segments below the filled circles can be reached only by bare strange stars. Asterisks are defined by the condition $R_{\mathrm{ms}}=R_{\mathrm{eq}}$ : below these asterisks in the high frequency region and between asterisks for $1.2 M_{\odot}$ case at intermediate rotation rate (thick part of the curve) $R_{\mathrm{ms}}<R_{\mathrm{eq}}$ and the ISCO is determined by the stellar radius $R_{\text {eq }}$. Dash-dotted line corresponds to the bare strange star of $1.2 M_{\odot}$. The difference with respect to the star with crust in the region $\sim 100-1100 \mathrm{kHz}$ is due to the fact that for bare strange stars the marginally stable orbit disappears at higher $\nu_{\text {rot }}$ and reappears at lower $\nu_{\text {rot }}$ leading to the $\nu_{\mathrm{ISCO}}$ larger than for SS with crust by $\sim 100 \mathrm{~Hz}$.

expect, $\nu_{\mathrm{ISCO}}$ is then equal to $\nu_{\mathrm{K}}$ since ISCO is determined by the equatorial radius of the star rotating with Keplerian frequency.

However it should be stressed that the lower limit on $\nu_{\text {ISCO }}$ consistent with the assumption that a marginally stable orbit exists (i.e. $R_{\mathrm{ms}}>R_{\mathrm{eq}}$ ) is significantly higher and is equal to $\sim 1.27 \mathrm{kHz}$ for SQM1 model and $1.1 \mathrm{kHz}$ for fine tuning of strange matter parameters, leading to the SQM2 EOS (Zdunik et al. 2000). For this last EOS $\nu_{\mathrm{ISCO}}<1 \mathrm{kHz}$ can be reached by bare SS for very rapid rotation. In this case the condition $R_{\mathrm{ms}}>R_{\text {eq }}$ eliminates rapidly rotating models with $\nu_{\mathrm{ISCO}}<1.1 \mathrm{kHz}$, and the condition $\nu_{\text {ISCO }}=1.07 \mathrm{kHz}$ with a non-zero gap can be fulfilled only by slowly rotating strange stars very close to maximum mass. Contrary to bare strange stars, when even for low mass stars rotating at the Keplerian limit, the frequency of the orbiting particle is limited by the marginally stable orbit and is approximately constant for a wide range of stellar masses (Zdunik \& Gourgoulhon 2001), for strange stars with a crust the marginally stable orbit does not exist at the Keplerian limit and the innermost stable orbit coincides with the radius of the star (except for the mass close to $M_{\max }$ ), which rapidly increases as the mass decreases, leading to the decrease of the frequency on ISCO.

\subsection{Parameters of the crust}

Two parameters of the crust, relevant for our considerations, are its baryon mass $M_{\mathrm{B} \text {,crust }}$ and its equatorial thickness $t_{\mathrm{eq}}$, defined in terms of the circumferential radius introduced in Sect. 2.3. In the static case these quantities will be denoted as $M_{\mathrm{B} \text {,crust }}^{0}$ and $t_{0}$, respectively. In the static case we will additionally consider crust contribution to the stellar gravitational mass $M_{\text {crust }}^{0}$. This quantity does not have a clear invariant meaning, reflecting the fact that gravitational energy cannot be localized in general relativity. Accordingly, $M_{\text {crust }}^{0}$ should be considered only as the difference between the total gravitational mass $M$ and the value at the interior/crust boundary of the metric coefficient $m(R)$ ("enclosed mass") which enters the Oppenheimer-Volkoff equations.

Let us start with the simplest case of a static strange star. For $M>M_{\odot}$, the ratio $t_{0} / R$ is small, and this enables us to derive approximate expressions resulting from the Oppenheimer-Volkoff equations of hydrostatic equilibrium

$$
\begin{aligned}
M_{\mathrm{crust}}^{0} & =\frac{4 \pi R^{4} P_{\mathrm{b}}}{G M}\left(1-\frac{2 G M}{R c^{2}}\right), \\
t_{0} & =\frac{\gamma}{\gamma-1} \frac{R^{2} c^{2}}{G M} \frac{P_{\mathrm{b}}}{\rho_{\mathrm{b}} c^{2}},
\end{aligned}
$$

where $\rho_{\mathrm{b}}$ and $P_{\mathrm{b}}$ are the density and pressure at the bottom of the crust, and $\gamma$ is the (average) adiabatic index of the outer crust.

For the BPS model (Baym et al. 1970) and $\rho_{\mathrm{b}}$ equal to the neutron drip density, we have $P_{\mathrm{b}}=7.8 \times$ $10^{29}$ dyn $\mathrm{cm}^{-2}, P_{\mathrm{b}} / \rho_{\mathrm{b}} c^{2}=0.002, \gamma=1.28$. This yields

$$
\begin{aligned}
t_{0} & =0.65 \frac{R_{6}^{2}}{M_{*}}\left(1-0.295 \frac{M_{*}}{R_{6}}\right) \mathrm{km} \\
M_{\text {crust }}^{0} & =3.7 \times 10^{-5} \frac{R_{6}^{4}}{M_{*}}\left(1-0.295 \frac{M_{*}}{R_{6}}\right) M_{\odot},
\end{aligned}
$$

where $R_{6}$ is the stellar radius in units $10^{6} \mathrm{~cm}$ and $M_{*}=$ $M / M_{\odot}$. The above formulae reproduce the exact results with an error lower than $6 \%$ in the case of $t_{0}$ and $4 \%$ for $M_{\text {crust }}$ for strange stars with mass between $1 M_{\odot}$ and $2 M_{\odot}$ (maximum mass). A comparison between approximate and exact values is displayed in Fig. 8.

Rotation will increase both the mass and the equatorial thickness of the crust. Results of Glendenning \& Weber (1992) for $t_{\text {eq }}$ as a function of $\nu_{\text {rot }}$ (at fixed value of $M_{\mathrm{B}}$ ) could be very precisely reproduced by a simple formula $t_{\mathrm{eq}}\left(\nu_{\mathrm{rot}}\right)=t_{0} \cdot\left[1+0.7\left(\nu_{\mathrm{rot}} / \nu_{\mathrm{K}}\right)^{2}\right]$, where $\nu_{\mathrm{K}}$ is the Keplerian (mass shedding) frequency of the strange star (Zdunik et al. 2000). A similar formula reproduces very well the rotational increase of the baryon mass of the crust. 

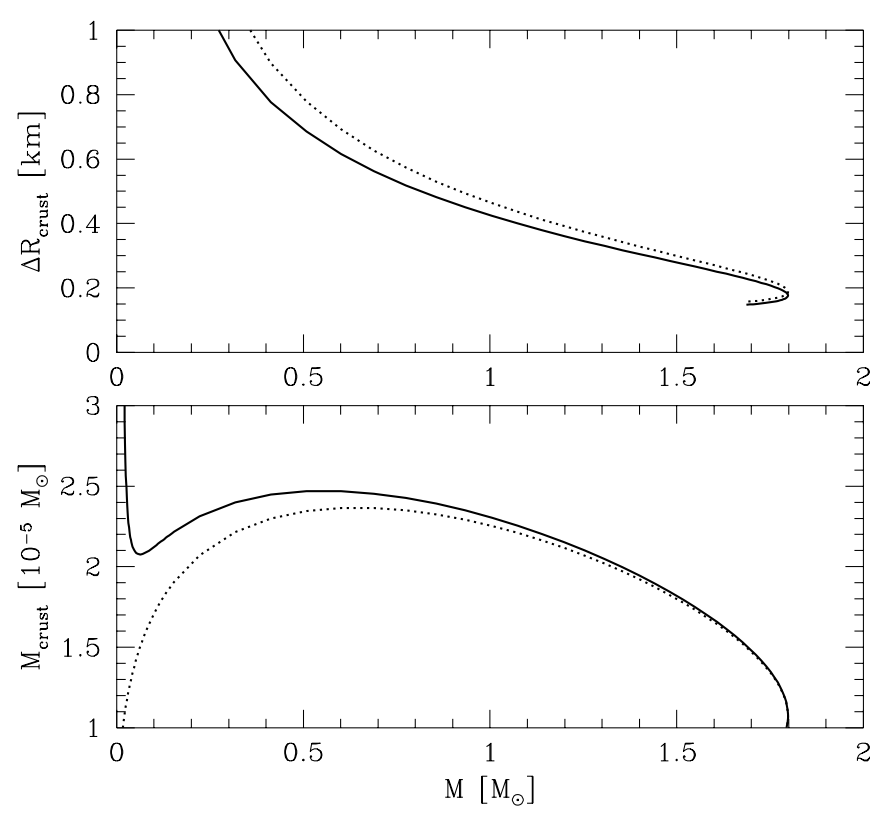

Fig. 8. Accuracy of the approximations (2) for the thickness and mass of the crust of a nonrotating strange star. Solid line: exact solution of the Oppenheimer-Volkoff equation; dotted line: approximation given by formula (2).

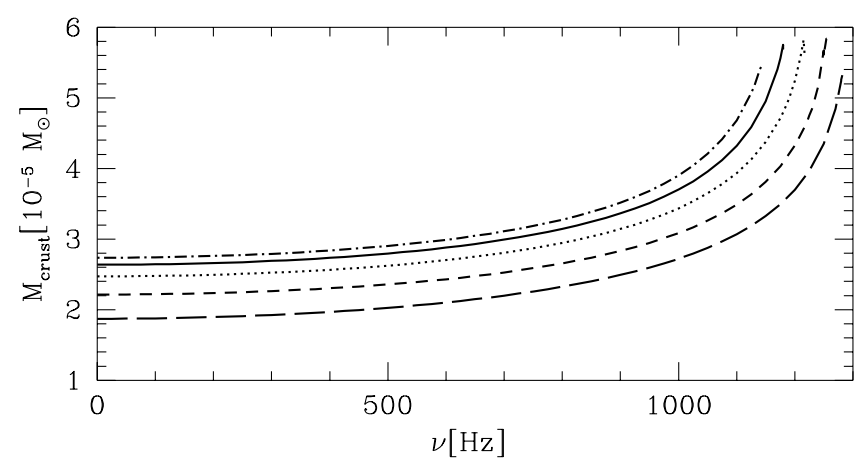

Fig. 9. Baryon mass of the crust as a function of rotational frequency, along sequences of constant baryon number. The gravitational masses $M$ of the nonrotating configuration of each sequence are $1.75,1.6,1.4,1.2,1 M_{\odot}$ (from bottom to top). Note that less massive stars have a larger crust (see also Fig. 8).

Exact results for the crust on the static and rotating strange stars are presented in Figs. 9 and 10. The mass of the crust which can be supported by the rotating strange star can even be 2.5 times larger than in the case of a nonrotating configuration. Another very important effect is a strong oblateness of the star, resulting in large $R_{\text {eq }}$. As a result, the crust at the equator is much thicker than at the pole (see Figs. 3 and 4).

The mass and thickness of the crust can be approximated by a quadratic function of rotation frequency up to about $500 \mathrm{~Hz}$. Thus the results of Glendenning \& Weber (1992) describe very well the parameters of the crust of the star rotating with intermediate frequencies (say below one half of the Keplerian one). For higher values of $\nu_{\text {rot }}$, both crust mass and equatorial thickness increase rapidly and we have to use a polynomial including terms of much
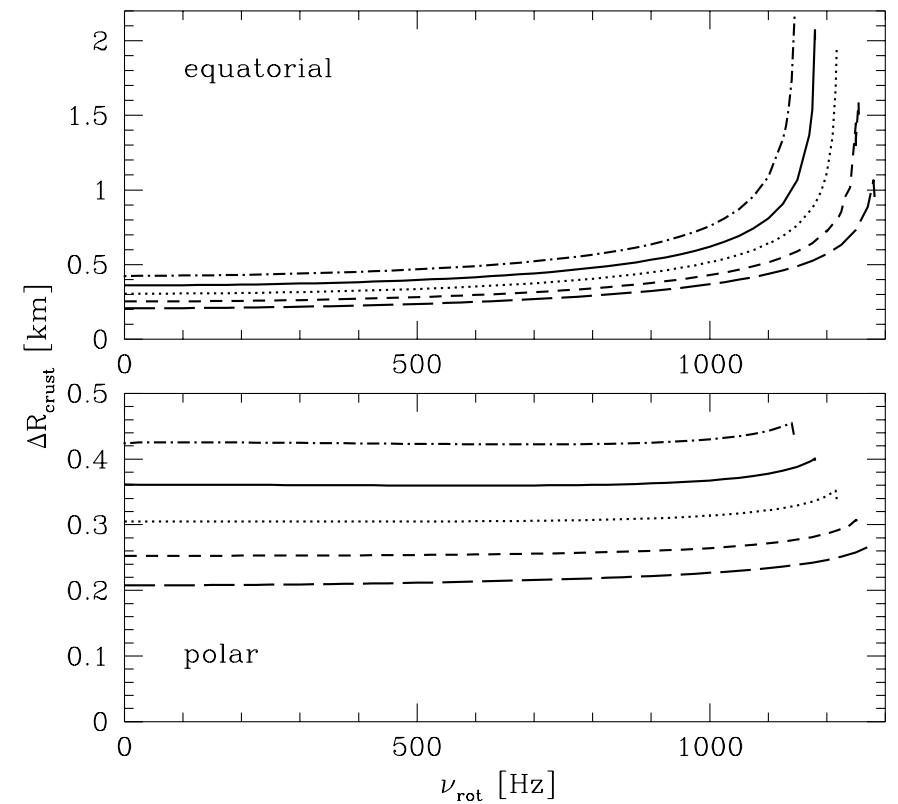

Fig. 10. Same as Fig. 9 but for the thickness of the crust (equatorial and polar). Note that the two plots are shown using different scales and that for a nonrotating star equatorial and polar thicknesses are equal.

higher (sixth to eighth) order in $\nu_{\text {rot }}$. The approximate formulae are found to be

$$
\begin{aligned}
& M_{\mathrm{B}, \text { crust }}=M_{\mathrm{B}, \text { crust }}^{0}\left(1+0.24 \nu_{\text {rot }, 3}^{2}+0.16 \nu_{\text {rot }, 3}^{8}\right), \\
& t_{\text {eq }}\left(\nu_{\text {rot }}\right)=t_{0} \cdot\left(1+0.4 \nu_{\text {rot }, 3}^{2}+0.3 \nu_{\text {rot }, 3}^{6}\right),
\end{aligned}
$$

where $\nu_{\text {rot }, 3}=\nu_{\text {rot }} / 10^{3} \mathrm{~Hz}$. Equation (3) works quite well nearly up to the Keplerian frequency, but the equatorial radius increases very rapidly as one approaches the Keplerian configuration. In view of this, Eq. (4) can be safely used below $1 \mathrm{kHz}$ (i.e. about $80 \%$ of the Keplerian value), where the change of the equatorial thickness is $\sim 100 \%$. The difference between our quadratic approximate formula and the fitting results of Glendenning \& Weber (1992) is a few percent.

Another interesting effect of rotation on the crust structure deserves comment. As Glendenning \& Weber (1992) pointed out, the polar thickness also increases (albeit weakly) due to the decrease in the gravitational force (resulting from the oblateness of mass distribution) at the pole. This effect is confirmed by our calculations (see bottom panel of Fig. 10).

\section{Deconfinement heating in spinning-down strange quark stars with crust}

The spin-down of a magnetized strange star implies a decrease of its oblateness. This process is particularly pronounced in the strange star crust. This change of shape is opposed by the elastic stress build-up in the Coulomb lattice of nuclei. The crust is relatively "soft" with respect to shear deformations, because its shear modulus constitutes only a few percent of the compression modulus 
(Haensel 1997; Haensel 2001). Therefore, in a zeroth approximation, the shape of the shrinking crust of a spinning-down strange star can be modeled by the equilibrium shape of a fluid. The spin-down implies some compression of the bottom matter elements of the crust, most pronounced in the equatorial belt. Consequently, as soon as the density exceeds the critical value $\rho_{\mathrm{b}}$, the bottom crust elements are engulfed by the SQM core. We note that if $\rho_{\mathrm{b}}=\rho_{\mathrm{ND}}$ then the absorption of crust matter is triggered by the neutron drip out of nuclei, while for $\rho_{\mathrm{b}}<\rho_{\mathrm{ND}}$ the absorption proceeds via quantum tunneling of nuclei through the Coulomb barrier, separating them from the SQM core (see Sect. 2.2).

A typical spin-down rate for a relatively young $1000 \mathrm{yr}$ old pulsar (like Crab) is $\dot{P} \sim 10^{-13} \mathrm{~s} / \mathrm{s}$, where $P$ is the pulsar period. Using the relation $\nu_{\text {rot }} \dot{\nu}_{\text {rot }}=-\dot{P} / P^{3}$, and expression (3) for the instantaneous baryon mass of the crust, we calculate the rate at which the baryon mass of the crust decreases due to the strange star spin-down:

$$
\begin{aligned}
\dot{M}_{\mathrm{B}, \text { crust }} \simeq & -9.6 \times 10^{14} \frac{M_{\mathrm{B}, \text { crust }}^{0}}{10^{-5} M_{\odot}}\left(\frac{P}{10 \mathrm{~ms}}\right)^{-3} \\
& \times \frac{|\dot{P}|}{10^{-13} \mathrm{~s} / \mathrm{s}} \mathrm{g} \mathrm{s}^{-1} .
\end{aligned}
$$

After crossing the SQM surface, nucleons (those in nuclei and dripped neutrons) dissolve into $u$ and $d$ quarks (via strong interactions), which will be followed by the strangeness-changing equilibration process $u+d \longrightarrow s+u$ via weak interaction. The whole process is expected to be strongly exothermic, with an energy release per unit absorbed baryon number $q_{\mathrm{dec}} \simeq 10-40 \mathrm{MeV}$, its specific value depending on the assumed SQM model (Farhi \& Jaffe 1984). Using Eq. (5), we estimate the total heating rate at the crust-SQM interface as

$$
\begin{aligned}
Q_{\mathrm{dec}} \simeq & 9 \times 10^{33} \cdot\left(\frac{P}{10 \mathrm{~ms}}\right)^{-3} \cdot \frac{|\dot{P}|}{10^{-13} \mathrm{~s} / \mathrm{s}} \\
& \times \frac{M_{\mathrm{B}, \text { crust }}^{0}}{10^{-5} M_{\odot}} \cdot \frac{q_{\mathrm{dec}}}{10 \mathrm{MeV}} \operatorname{erg~s}^{-1} .
\end{aligned}
$$

The typical deconfinement heating, estimated above, may therefore exceed the neutrino losses (luminosity) from the SQM core of a 1000 years old (or older) strange star with a crust, $L_{\nu} \sim 10^{31}(R / 10 \mathrm{~km})^{3}\left(T / 10^{7} \mathrm{~K}\right)^{6} \mathrm{erg} / \mathrm{s}$, and therefore could significantly influence (namely - delay) the cooling of this compact object (Yuan \& Zhang 1999). We mention that the deconfinement heating, described above, is much more efficient than the roto-chemical heating, resulting from deviations of the composition of the SQM core of a spinning-down strange star from beta equilibrium and studied in Cheng \& Dai (1996).

\section{Summary and conclusion}

We calculated exact 2-D models of stationary rotating strange stars of masses $1.2-1.75 M_{\odot}$, covered with a crust of normal matter, up to the Keplerian limit. Despite its low mass $\left(\sim 10^{-5} M_{\odot}\right)$ and small thickness (a few hundred meters), the solid crust plays an important role for these hypothetical objects. We considered sequences of uniformly rotating strange stars of a fixed baryon mass, and increasing values of angular momentum $J$, and we found that due to the large deformation near the Keplerian limit, the decrease of the maximum frequency, compared to that for bare strange stars, is very small. Close to the Keplerian limit, the stellar moment of inertia increases rapidly with rotation frequency, and this results in a weak dependence of the maximum frequency of uniform rotation, $\nu_{\max }$, on the value of $J_{\max }$, which in the presence of the crust is lower by as much as twenty percent compared to that of bare strange stars. On the contrary, the effect of the presence of the crust on the properties of the innermost stable circular stable orbit is important, and consists of about fifteen percent increase in $\nu_{\mathrm{ISCO}}$ at the Keplerian limit, as well as the disappearance of the gap between the ISCO and stellar surface for a broad range of stellar masses and rotation frequencies. The solid crust also lowers by as much as twenty percent the maximum $T / W$ ratio which can be reached by a rotating strange star, from $0.23-0.25$ to $0.18-0.20$.

Both the mass of the crust and its equatorial thickness increase with the rotation frequency. We have shown that this increase is very well approximated by simple and precise analytical formulae up to $80 \%$ of the Keplerian frequency. For a rotation frequency below one third of the maximum, the increase with respect to the static case is quadratic in the rotation frequency. However, close to the Keplerian limit, the dependence is much more rapid, which is consistent with the characteristic behavior of $J\left(\nu_{\text {rot }}\right)$, mentioned in the previous paragraph.

Acknowledgements. This research was partially supported by the KBN grant No. 5P03D.020.20. The numerical calculations have been performed on computers purchased thanks to a special grant from the SPM and SDU departments of CNRS. The main results reported in the present paper were obtained during a visit of one of the authors (J.L.Z) at DARC, Observatoire de Paris-Meudon, within the framework of the CNRS/PAN program Jumelage Astrophysique.

\section{References}

Alcock, C., Farhi, C. E., \& Olinto, A. 1986, ApJ, 310, 261

Baym, G., Pethick, C., \& Sutherland, P. 1971, ApJ, 170, 299

Bombaci, I. 1997, Phys. Rev. C, 55, 1587

Bonazzola, S., \& Gourgoulhon, E. 1994, Class. Quantum Grav., 11,1775

Bonazzola, S., Gourgoulhon, E., \& Marck, J. A. 1998, Phys. Rev. D, 58, 104020

Bulik, T., Gondek-Rosińska, D., \& Kluźniak, W. 1999, A\&A, 344, L71

Cheng, K. S., \& Dai, Z. G. 1996, ApJ, 468, 819

Cheng, K. S., Dai, Z. G., Wei, D. M., \& Lu, T. 1998, Science, 280, 407

Cook, G. B., Shapiro, S. L., \& Teukolsky, S. A. 1994, ApJ, 424, 823

Dai, Z. G., \& Lu, T. 1998, Phys. Rev. Lett., 81, 4301 
Farhi, E., \& Jaffe, R. L. 1984, Phys. Rev. D, 30, 2379

Glendenning, N. K., \& Weber, F. 1992, ApJ, 400, 647

Gondek-Rosińska, D., Bulik, T., Zdunik, L., et al. 2000, A\&A, 363, 1005

Gourgoulhon, E., \& Bonazzola, S. 1994, Class. Quantum Grav., 11, 443

Gourgoulhon, E., Haensel, P., Livine, R., et al. 1999, A\&A, 349,851

Haensel, P. 1997, in Relativistic Gravitation and Gravitational Radiation, ed. J.-A. Marck, \& J.-P. Lasota (Cambridge University Press), 129

Haensel, P. 2001, in Physics of Neutron Star Interiors, ed. D. Blaschke, N. K. Glendenning, \& A. Sedrakian (Springer Verlag), in press

Haensel, P., Zdunik, J. L., \& Schaeffer, R. 1986, A\&A, 160, 121

Haensel, P., \& Zdunik, J. L. 1991, Nucl. Phys. B (Proc. Suppl.), 24B, 139

Hartle, J. B. 1967, ApJ, 150, 1005

Huang, Y. F., \& Lu, T. 1997, A\&A, 325, 189
Kaaret, P., Ford, E. C., \& Chen, K. 1997, ApJL, 480, L27

Kaaret, P., Piraino, S., Bloser, P. F., et al. 1999, ApJL, 520, L37

Li, X.-D., Bombaci, I., Dey, M., Dey, J., \& van den Heuvel, E. P. J. 1999, Phys. Rev. Lett., 83, 3776

Madsen, J. 1999, in Hadrons in Dense Matter and Hadrosynthesis, Lecture Notes in Physics, ed. J. Cleymans (Springer-Verlag), 162

Miralda-Escudé, J., Haensel, P., \& Paczyński, B. 1990, ApJ, 362,572

Shapiro, S. L., \& Zane, S. 1998, ApJS, 117, 531

Shibata, M., \& Sasaki, M. 1998, Phys. Rev. D, 58, 104011

Stergioulas, N., Kluźniak, W., \& Bulik, T. 1999, A\&A, 352, L116

Witten, E. 1984, Phys. Rev. D, 30, 272

Yuan, Y. F., \& Zhang, J. L. 1999, A\&A, 344, 371

Zdunik, J. L., \& Gourgoulhon, E. 2001, Phys. Rev. D, 63, 087501

Zdunik, J. L., Haensel, P., Gondek-Rosińska, \& Gourgoulhon, E. 2000, A\&A, 356, 612 